\title{
A Note on the Stability of Some Functional Equations on Certain Groupoids
}

\author{
Jedsada Senasukh and Satit SAejunG*
}

ABSTRACT. In this paper, we show that the stability of Cauchy set-valued functional equations and of Jensen setvalued functional equations can be derived from the stability of the corresponding equations in single-valued version.

Keywords: Stability, Cauchy set-valued functional equation, Jensen set-valued functional equation, fixed point method.

2010 Mathematics Subject Classification: 39B52, 39B82, 54C60, 47H10

\section{INTRODUCTION}

In the sequel, $\mathbb{N}, \mathbb{N}_{0}, \mathbb{R}$ and $\mathbb{R}_{+}$denote the set of positive integers, the set of nonnegative integers, the set of real numbers, and the set of nonnegative real numbers, respectively. For two nonempty sets $X$ and $Y, Y^{X}$ denotes the class of all functions from $X$ into $Y$.

Given a groupoid $(G, \circ)$, the binary operation $\circ$ is square-symmetric if

$$
2(a \circ b)=2 a \circ 2 b \text { for all } a, b \in G \text {. }
$$

Here $2 a:=a \circ a$ for all $a \in G$. It is clear that every commutative semigroup is a squaresymmetric groupoid but the converse is not true. (In fact, let $G:=\mathbb{N}$ be equipped with a binary operation $a \circ b:=a+2 b$ for all $a, b \in G$. Then $(G, \circ)$ is a square-symmetric groupoid and $\circ$ is not associative.) Recall that a groupoid $(G, \circ)$ is divisible if for each $a \in G$ there exists a unique element $a^{\prime} \in G$ such that $2 a^{\prime}=a$. For convenience, we will write $\frac{a}{2}:=a^{\prime}$ or $\frac{1}{2} a:=a^{\prime}$. To simplify the notation, for each $a$ in a groupoid $G:=(G, \circ)$ and each $n \in \mathbb{N}_{0}$, we write $2^{0} a:=a$ and $2^{n+1} a:=2\left(2^{n} a\right)$. If, in addition, $G$ is divisible, then we also write $\frac{a}{2^{0}}:=a$ and $\frac{a}{2^{n+1}}:=\frac{1}{2}\left(\frac{a}{2^{n}}\right)$ all $n \in \mathbb{N}_{0}$.

Lemma 1.1. Suppose that $(G, \circ)$ is a square-symmetric groupoid. Then, the following assertions are true.

(1) $2^{n}(a \circ b)=2^{n} a \circ 2^{n} b$ for all $a, b \in G$ and all $n \in \mathbb{N}_{0}$.

(2) $\frac{1}{2^{n}}(a \circ b)=\frac{a}{2^{n}} \circ \frac{b}{2^{n}}$ for all $a, b \in G$ and all $n \in \mathbb{N}_{0}$ provided that $G$ is divisible.

From now on, we assume that:

- $G:=(G, \circ)$ and $H:=(H, *)$ are square-symmetric groupoids;

- $(H, d)$ is a complete metric space such that $*$ is continuous, that is, $\lim _{k \rightarrow \infty} d\left(u_{k} * v_{k}, u * v\right)=0$ whenever $\lim _{k \rightarrow \infty} d\left(u_{k}, u\right)=\lim _{k \rightarrow \infty} d\left(v_{k}, v\right)=0$;

- $\varphi: G \times G \rightarrow \mathbb{R}_{+}$is a function.

Received: 25.02.2020; Accepted: 31.05.2020; Published Online: 31.05.2020

*Corresponding author: Satit Saejung; saejung@kku.ac.th

DOI: $10.33205 / \mathrm{cma} .729765$ 
To study the stability of a Cauchy functional equation, we define the following class:

$$
\mathscr{C}(G, H, \varphi):=\left\{f \in H^{G}: d(f(x \circ y), f(x) * f(y)) \leq \varphi(x, y) \text { for all } x, y \in G\right\} .
$$

Now, we introduce the stability concept of a functional equation as follows.

Definition 1.2. The Cauchy functional equation from $G$ into $H$ is said to be $\varphi$-stable if the class $\mathscr{C}(G, H, \varphi)$ satisfies the following property:

there exists a function $\Phi \in \mathbb{R}_{+}^{G}$ such that for every $f \in \mathscr{C}(G, H, \varphi)$ there exists a unique function $F \in \mathscr{C}(G, H, \mathbf{0})$, that is, $F(x \circ y)=F(x) * F(y)$ for all $x, y \in G$, such that

$$
d(F(x), f(x)) \leq \Phi(x) \text { for all } x \in G .
$$

In this case, we also say that the class $\mathscr{C}(G, H, \varphi)$ is $\varphi$-stable with respect to $\Phi$.

In 1940, Ulam [17] proposed a problem concerning the stability of the Cauchy functional equation from a group $G$ into a metric group $H$. A year later, Hyers [10] was the first mathematician who answered Ulam's problem if $G$ and $H$ are Banach spaces. Many generalizations of Hyers' result have been studied [1, 8, 9, 15]. Inspired by the notion of square-symmetry, Páles et al. [12] and Kim [11] proved some stability results of the Cauchy functional equation from square-symmetric groupoids into metric square-symmetric groupoids based on the control function proposed by Găvruţa [9].

We now recall the following conditions given by $\operatorname{Kim}[11]$. A triplet $(G, H, \varphi)$ satisfies

Condition (K1): if the following two conditions hold:

(K1a) $H:=(H, *)$ is divisible;

(K1b) there exists a real number $\gamma>0$ such that $d\left(\frac{u}{2}, \frac{v}{2}\right) \leq \gamma d(u, v)$ for all $u, v \in H$ and $\widetilde{\varphi}(x, y):=\sum_{k=0}^{\infty} \gamma^{k} \varphi\left(2^{k} x, 2^{k} y\right)<\infty$ for all $x, y \in G ;$

Condition (K2): if the following two conditions hold:

(K2a) $G:=(G, \circ)$ is divisible;

(K2b) there exists a real number $\gamma>0$ such that $d(2 u, 2 v) \leq \gamma d(u, v)$ for all $u, v \in H$ and $\widetilde{\varphi}(x, y):=\sum_{k=1}^{\infty} \gamma^{k} \varphi\left(\frac{x}{2^{k}}, \frac{y}{2^{k}}\right)<\infty$ for all $x, y \in G$.

The following stability result was proved by Kim [11].

Theorem K. If $(G, H, \varphi)$ satisfies either Condition (K1) or Condition (K2), then the Cauchy functional equation from $G$ into $H$ is $\varphi$-stable. Moreover, the class $\mathscr{C}(G, H, \varphi)$ is $\varphi$-stable with respect to $\Phi$, where

$$
\Phi(x):= \begin{cases}\gamma \widetilde{\varphi}(x, x) & \text { if }(G, H, \varphi) \text { satisfies Condition (K1); } \\ \frac{1}{\gamma} \widetilde{\varphi}(x, x) & \text { if }(G, H, \varphi) \text { satisfies Condition (K2); }\end{cases}
$$

for all $x \in X$.

The authors were informed by the referee that Theorem $\mathrm{K}$ with Condition (K1) is related to the result of Forti [7].

Remark 1.3. According to Theorem $\mathrm{K}$, for each $f \in \mathscr{C}(G, H, \varphi)$, the function $F \in \mathscr{C}(G, H, \mathbf{0})$ is uniquely determined by

$$
F(x)= \begin{cases}\lim _{k \rightarrow \infty} \frac{1}{2^{k}} f\left(2^{k} x\right) & \text { if }(G, H, \varphi) \text { satisfies Condition (K1); } \\ \lim _{k \rightarrow \infty} 2^{k} f\left(\frac{x}{2^{k}}\right) & \text { if }(G, H, \varphi) \text { satisfies Condition (K2); }\end{cases}
$$

for all $x \in X$. 
A concept of set-valued functions in Banach spaces have been developed in the last decades. The result concerning the set-valued functional equation (a functional equation whose solutions are set-valued functions) seems to be pioneered by Aumann [3] and Debreu [2]. We recall the following: Suppose that $X:=(X,\|\cdot\|)$ is a real normed space. We define

$$
\begin{aligned}
\mathcal{P}(X) & :=\{A: A \text { is a subset of } X\} ; \\
\operatorname{BCC}(X) & :=\{A \in \mathcal{P}(X) \backslash\{\varnothing\}: A \text { is bounded, closed, and convex }\} .
\end{aligned}
$$

For each $A, B \in \mathrm{BCC}(X)$ and $\lambda \in \mathbb{R}$, we define:

- $A \oplus B:=\operatorname{cl}\{a+b: a \in A$ and $b \in B\}$ (cl := the closure);

- $\lambda A:=\{\lambda a: a \in A\}$;

- $\mathcal{H}(A, B):=\max \left\{\sup _{a \in A} \inf _{b \in B}\|a-b\|, \sup _{b \in B} \inf _{a \in A}\|a-b\|\right\}$;

- $\operatorname{diam} A:=\sup _{a, a^{\prime} \in A}\left\|a-a^{\prime}\right\|$.

It is worth mentioning that in the definition of $\oplus$ the closure is needed because $\{a+b: a \in$ $A$ and $b \in B\}$ does not necessarily belong to $\mathrm{BCC}(X)$ if $A, B \in \mathrm{BCC}(X)$ [14, Theorem 2.1].

By using the fixed point alternative method proposed by Diaz and Magolis [6], Park et al. [13] proved some stability results of the Cauchy functional equations from a real normed space $X$ into a complete metric commutative semigroup $(\operatorname{BCC}(Y), \oplus)$, where $Y$ is a Banach space.

In this paper, we first show that the results of Park et al. [13] for the Cauchy functional equation from a real normed space $X$ into $(\operatorname{BCC}(Y), \oplus)$ (where $Y$ is a Banach space) is a consequence of Theorem K. In addition, inspired by the work of Kim [11], we prove the stability results of the Jensen functional equations by using a modified Brzdęk's fixed point theorem [16]. We also point out that the stability result of Jensen set-valued functional equations can be derived from that of the corresponding on certain groupoids. Roughly speaking, we obtain the stability result of the Cauchy "set-valued" functional equation and that of the Jensen "set-valued" functional equation from the corresponding results of the "single-valued" version.

\section{MAin RESULtS}

\subsection{Stability of Cauchy set-valued functional equations via that of Cauchy single-valued} functional equations. We first recall the following properties.

Lemma 2.1. Let $X$ be a real normed space. Suppose that $A, B, C, D \in \mathrm{BCC}(X)$ and $\lambda, \mu \in \mathbb{R}_{+}$. Then, following assertions are true.

(a) $A \oplus B \in \mathrm{BCC}(X)$ and $\lambda A \in \operatorname{BCC}(X)$.

(b) $\lambda A \oplus \lambda B=\lambda(A \oplus B)$ and $(\lambda+\mu) A=\lambda A \oplus \mu A$.

(c) $\mathcal{H}(A \oplus C, B \oplus D) \leq \mathcal{H}(A, B)+\mathcal{H}(C, D)$.

(d) $|\operatorname{diam} A-\operatorname{diam} B| \leq 2 \mathcal{H}(A, B)$.

(e) $\mathcal{H}(\lambda A, \lambda B)=\lambda \mathcal{H}(A, B)$.

(f) If $X$ is a Banach space, then $(\mathrm{BCC}(X), \oplus, \mathcal{H})$ is a complete metric divisible commutative semigroup (see [5]).

We obtain the following proposition as a consequence of Lemma 2.1(d).

Proposition 2.2. Suppose that $X, Y$ are two real normed spaces and $f: X \rightarrow \mathrm{BCC}(Y)$ is a function. Then, the following assertions are true.

(i) If $\lim _{k \rightarrow \infty} \operatorname{diam} f\left(2^{k} x\right) / 2^{k}=0$ for all $x \in X$, then $F(x):=\lim _{k \rightarrow \infty} f\left(2^{k} x\right) / 2^{k}$ is singlevalued for all $x \in X$.

(ii) If $\lim _{k \rightarrow \infty} \operatorname{diam} 2^{k} f\left(x / 2^{k}\right)=0$ for all $x \in X$, then $F(x):=\lim _{k \rightarrow \infty} 2^{k} f\left(x / 2^{k}\right)$ is singlevalued. 
By using Lemma 2.1(f), we obtain the main results of Park et al. [13, Theorems 2.2 and 2.3] as a consequence of Theorem $\mathrm{K}$.

Corollary 2.3. Suppose that $X$ is a real normed space and $Y$ is a real Banach space. If there exists $L \in(0,1)$ and one of the following conditions is satisfied:

(1) $\varphi(x, y) \leq 2 L \varphi\left(\frac{x}{2}, \frac{y}{2}\right)$ for all $x, y \in X$;

(2) $\varphi(x, y) \leq \frac{L}{2} \varphi(2 x, 2 y)$ for all $x, y \in X$,

then the Cauchy functional equation is $\varphi$-stable in the class of set-valued functions $\mathscr{C}(G, H, \varphi)$. Moreover, the class $\mathscr{C}(G, H, \varphi)$ is $\varphi$-stable with respect to $\Phi$, where

$$
\Phi(x):= \begin{cases}\frac{1}{2-2 L} \varphi(x, 0) & \text { if (1) holds; } \\ \frac{L}{2-2 L} \varphi(x, 0) & \text { if (2) holds; }\end{cases}
$$

for all $x \in X$. In addition, if $f \in \mathscr{C}(X, \operatorname{BCC}(Y), \varphi)$ and there exist positive real numbers $M$ and $\alpha$ such that: $\operatorname{diam} f(x) \leq M\|x\|^{\alpha}$ for all $x \in X$, where $\alpha \in(0,1)$ if $(1)$ holds; or $\alpha \in(1, \infty)$ if $(2)$ holds, then the set-valued function $F$ given by Remark 1.3 is single-valued.

\subsection{Stability of the Jensen functional equations and some results for Jensen "multi-valued"}

functional equations. Throughout this subsection, we assume that a square-symmetric groupoid $G:=(G, \circ)$ has an identity $e_{G}$. We define the following two classes of functions (we remark that in the second class the divisibility of $G$ is required):

$$
\begin{aligned}
& \mathscr{J}_{1}(G, H, \varphi):=\left\{\begin{array}{c}
f(2 f(x \circ y), f(2 x) * f(2 y)) \leq \varphi(x, y) \text { for all } x, y \in G \text { and } \\
f\left(e_{G}\right) * f(x)=f(x)=f(x) * f\left(e_{G}\right) \text { for all } x \in G
\end{array}\right\}, \\
& \mathscr{J}_{2}(G, H, \varphi):=\left\{\begin{array}{cc}
f \in H^{G}: & d\left(2 f\left(\frac{x \circ y}{2}\right), f(x) * f(y)\right) \leq \varphi(x, y) \text { for all } x, y \in G \text { and } \\
f\left(e_{G}\right) * f(x)=f(x)=f(x) * f\left(e_{G}\right) \text { for all } x \in G
\end{array}\right\} .
\end{aligned}
$$

We now introduce the stability notion of the Jensen functional equations as follows.

Definition 2.4. The Jensen functional equation of the first (second, resp.) kind from $G$ into $H$ is said to be $\varphi$-stable if the class $\mathscr{J}_{1}(G, H, \varphi)\left(\mathscr{J}_{2}(G, H, \varphi)\right.$, resp.) satisfies the following property:

there exist a function $\Phi \in \mathbb{R}_{+}^{G}$ such that for every $f \in \mathscr{J}_{1}(G, H, \varphi)\left(f \in \mathscr{J}_{2}(G, H, \varphi)\right.$, resp.) there exists a unique function $F \in \mathscr{J}_{1}(G, H, \mathbf{0})\left(F \in \mathscr{J}_{2}(G, H, \mathbf{0})\right.$, resp. $)$, that is, $2 F(x \circ y)=F(2 x) * F(2 y)$ for all $x, y \in G\left(2 F\left(\frac{x \circ y}{2}\right)=F(x) * F(y)\right.$ for all $x, y \in G$, resp.), such that

$$
d(F(x), f(x)) \leq \Phi(x) \text { for all } x \in G .
$$

In this case, we say that the class $\mathscr{J}_{1}(G, H, \varphi)\left(\mathscr{J}_{2}(G, H, \varphi)\right.$, resp.) is $\varphi$-stable with respect to $\Phi$.

Brzdęk [4] proved a fixed point theorem for the stability result of the Cauchy functional equation on a commutative semigroup. Later, Saejung and Senasukh [16] modified Brzdęk's fixed point theorem and also proved some stability results of some functional equations on restricted domains. The proof of the following fixed point theorem is similar to the one given by Saejung and Senasukh [16], so it is omitted.

Theorem 2.5. Suppose that $X$ is a nonempty set and $(Y, d)$ is a complete metric space. Suppose that $\mathcal{T}: Y^{X} \rightarrow Y^{X}$ and $\theta: X \rightarrow X$ are mappings and there exists $\alpha \in \mathbb{R}^{+}$such that

$$
d((\mathcal{T} \xi)(x),(\mathcal{T} \mu)(x)) \leq \alpha d(\xi(\theta(x)), \mu(\theta(x))) \quad \text { for all } \xi, \mu \in Y^{X} \text { and all } x \in X
$$


If there exists a mapping $\varphi: X \rightarrow Y$ such that

$$
\varepsilon^{*}(x):=\sum_{k=0}^{\infty} d\left(\left(\mathcal{T}^{k} \varphi\right)(x),\left(\mathcal{T}^{k+1} \varphi\right)(x)\right)<\infty \quad \text { for all } x \in X,
$$

then $\mathcal{T}$ has a fixed point $\psi \in Y^{X}$ such that

$$
d(\psi(x), \varphi(x)) \leq \varepsilon^{*}(x) \quad \text { for all } x \in X .
$$

Moreover, $\psi(x)=\lim _{k \rightarrow \infty}\left(\mathcal{T}^{k} \varphi\right)(x)$ for all $x \in X$.

As a consequence of our fixed point theorem (Theorem 2.5), we first obtain the following stability result of the Jensen functional equation of the first kind.

Theorem 2.6. If $(G, H, \varphi)$ satisfies either Condition (K1) or Condition (K2), then the Jensen functional equation from $G$ into $H$ is $\varphi$-stable. Moreover, the class $\mathscr{J}_{1}(G, H, \varphi)$ is $\varphi$-stable with respect to $\Phi$, where

$$
\Phi(x):= \begin{cases}\gamma \widetilde{\varphi}\left(x, e_{G}\right) & \text { if }(G, H, \varphi) \text { satisfies Condition }(K 1) \\ \frac{1}{\gamma} \widetilde{\varphi}\left(x, e_{G}\right) & \text { if }(G, H, \varphi) \text { satisfies Condition }(K 2)\end{cases}
$$

for all $x \in X$.

Proof. We first suppose that $(G, H, \varphi)$ satisfies Condition (K1). Let $f \in \mathscr{J}_{1}(G, H, \varphi)$ be given. Set $X:=(G,+)$ and $Y:=(H, *, d)$. Define mappings $\mathcal{T}: Y^{X} \rightarrow Y^{X}$ and $\theta: X \rightarrow X$ by

$$
(\mathcal{T} \xi)(x):=\frac{1}{2} \xi(2 x) \text { and } \quad \theta(x):=2 x
$$

for all $\xi \in Y^{X}$ and all $x \in X$. We also let $\alpha:=\gamma$. For each $\xi, \mu \in Y^{X}$ and each $x \in X$, we see that

$$
d((\mathcal{T} \xi)(x),(\mathcal{T} \mu)(x)) \leq \gamma d(\xi(2 x), \mu(2 x))=\alpha d(\xi(\theta(x)), \mu(\theta)(x)) .
$$

We show that the following inequality holds for all $n \in \mathbb{N}_{0}$;

$$
d\left(2\left(\mathcal{T}^{n} f\right)(x \circ y),\left(\mathcal{T}^{n} f\right)(2 x) *\left(\mathcal{T}^{n} f\right)(2 y)\right) \leq \gamma^{n} \varphi\left(2^{n} x, 2^{n} y\right) \quad \text { for all } x, y \in X .
$$

The case $n=0$ is trivially true. Suppose that (2.2) is valid for some $k \in \mathbb{N}_{0}$. Let $x, y \in X$. Because of Condition (K1), we have

$$
\begin{aligned}
& d\left(2\left(\mathcal{T}^{k+1} f\right)(x \circ y),\left(\mathcal{T}^{k+1} f\right)(2 x) *\left(\mathcal{T}^{k+1} f\right)(2 y)\right) \\
& =d\left(\frac{1}{2}\left(2\left(\mathcal{T}^{k} f\right)\right)(2 x \circ 2 y), \frac{1}{2}\left(\mathcal{T}^{k} f\right)(2(2 x)) * \frac{1}{2}\left(\mathcal{T}^{k} f\right)(2(2 y))\right) \\
& \leq \gamma\left(\gamma^{k} \varphi\left(2^{k}(2 x), 2^{k}(2 y)\right)=\gamma^{k+1} \varphi\left(2^{k+1} x, 2^{k+1} y\right) .\right.
\end{aligned}
$$

This shows that (2.2) is valid for all $n \in \mathbb{N}_{0}$ for all $x \in X$.

Let $k \in \mathbb{N}_{0}$ and $x \in X$ be given. We have

$$
\varepsilon^{*}(x):=\sum_{k=0}^{\infty} d\left(\left(\mathcal{T}^{k} f\right)(x),\left(\mathcal{T}^{k+1} f\right)(x)\right) \leq \gamma \sum_{k=0}^{\infty} \gamma^{k} \varphi\left(2^{k} x, e_{G}\right)<\infty .
$$

It follows from Theorem 2.5 that $\mathcal{T}$ has a fixed point $F: X \rightarrow Y$ such that

$$
d(F(x), f(x)) \leq \varepsilon^{*}(x) \leq \gamma \widetilde{\varphi}\left(x, e_{G}\right) \quad \text { for all } x \in X .
$$

Moreover, $F(x)=\lim _{k \rightarrow \infty}\left(\mathcal{T}^{k} f\right)(x)=\lim _{k \rightarrow \infty} \frac{1}{2^{k}} f\left(2^{k} x\right)$ for all $x \in X$. The continuity of $*$ and (2.2) imply that

$$
2 F(x \circ y)=F(2 x) * F(2 y) \quad \text { for all } x, y \in X \text {. }
$$


We finally prove the uniqueness part. To prove this, suppose that there exist a function $\widetilde{F} \in \mathscr{J}_{1}(G, H, \mathbf{0})$ and a constant $C>0$ such that

$$
d(\widetilde{F}(x), f(x)) \leq C \widetilde{\varphi}\left(x, e_{G}\right) \text { for all } x \in X .
$$

For each $k \in \mathbb{N}_{0}$ and each $x \in X$, we first note that

$$
F(x)=\frac{1}{2^{k}} F\left(2^{k} x\right) \quad \text { and } \quad \widetilde{F}(x)=\frac{1}{2^{k}} \widetilde{F}\left(2^{k} x\right) .
$$

We consider

$$
\begin{aligned}
d(F(x), \widetilde{F}(x)) & \leq d\left(\frac{1}{2^{k}} F\left(2^{k} x\right), \frac{1}{2^{k}} f\left(2^{k} x\right)\right)+d\left(\frac{1}{2^{k}} \widetilde{F}\left(2^{k} x\right), \frac{1}{2^{k}} f\left(2^{k} x\right)\right) \\
& \leq \gamma^{k}(\gamma+C) \widetilde{\varphi}\left(2^{k} x, e_{G}\right)=(\gamma+C) \sum_{i=k}^{\infty} \gamma^{i} \varphi\left(2^{i} x, e_{G}\right) .
\end{aligned}
$$

By letting $k \rightarrow \infty$, we get $\widetilde{F}(x)=F(x)$.

With the similar method, we can prove the result if $(G, H, \varphi)$ satisfies Condition (K2).

Remark 2.7. According to Theorem 2.6, for each $f \in \mathscr{J}_{1}(G, H, \varphi)$ the function $F \in \mathscr{J}_{1}(G, H, \mathbf{0})$ is uniquely determined by $f$ as in Remark 1.3.

The following two examples show that the bound $\Phi(x)$ in Theorem 2.6 is optimal in some particular cases.

Example 2.8. Let $G:=\left(\mathbb{R}_{+},+\right)$and $H:=(\mathbb{R},+, d)$, where $d(x, y):=|x-y|$ for all $x, y \in H$, and $f: G \rightarrow H$ be defined by $f(x):=\sqrt{x}$ for all $x \in G$. We see that

$$
d(2 f(x \circ y), f(2 x) * f(2 y))=|2 \sqrt{x+y}-\sqrt{2 x}-\sqrt{2 y}|:=\varphi(x, y) \quad \text { for all } x, y \in G .
$$

Then $f \in \mathscr{J}_{1}(G, H, \varphi)$ and $(G, H, \varphi)$ satisfies Condition (K1) with $\gamma=\frac{1}{2}$. For each $x \in G$, we also note that

$$
\widetilde{\varphi}(x, 0):=\sum_{k=0}^{\infty} \frac{1}{2^{k}} \varphi\left(2^{k} x, 0\right)=\sum_{k=0}^{\infty} 2^{-k / 2} \varphi(x, 0)=(2+\sqrt{2}) \varphi(x, 0) .
$$

Note that $F(x)=0$ and $\Phi(x)=\gamma \widetilde{\varphi}(x, 0)=\sqrt{x}=f(x)$ for all $x \in G$.

Example 2.9. Let $G:=(\mathbb{R},+), H:=(\mathbb{R},+, d)$ (where $d$ is defined as in Example 2.8), and $f: G \rightarrow H$ be defined by $f(x):=x^{2}$ for all $x \in G$. We see that

$$
d(2 f(x \circ y), f(2 x) * f(2 y))=2(x-y)^{2}:=\varphi(x, y) \quad \text { for all } x, y \in G .
$$

Then $f \in \mathscr{J}_{2}(G, H, \varphi)$ and $(G, H, \varphi)$ satisfies Condition (K2) with $\gamma=2$. For each $x \in G$, we note that

$$
\widetilde{\varphi}(x, 0)=\sum_{k=1}^{\infty} 2^{k} \varphi\left(\frac{x}{2^{k}}, 0\right)=\sum_{k=1}^{\infty} 2^{-k} \varphi(x, 0)=\varphi(x, 0) .
$$

Note that $F(x)=0$ and $\Phi(x)=\frac{1}{\lambda} \widetilde{\varphi}(x, 0)=\frac{1}{2} \varphi(x, 0)=x^{2}=f(x)$ for all $x \in G$.

By using Theorem 2.6, we obtain the following stability result given by Park et. al. [13, Theorems 3.4 and 3.5] as a consequence.

Corollary 2.10. Suppose that $X$ is a real normed space and $Y$ is a real Banach space. If there exists $L \in(0,1)$ and one of the following conditions is satisfied:

(1) $\varphi(x, y) \leq 2 L \varphi\left(\frac{x}{2}, \frac{y}{2}\right)$ for all $x, y \in X$;

(2) $\varphi(x, y) \leq \frac{L}{2} \varphi(2 x, 2 y)$ for all $x, y \in X$, 
then the Jensen functional equation is $\varphi$-stable in the class of set-valued functions $\mathscr{J}_{2}(X, \operatorname{BCC}(Y), \varphi)$. Moreover, the class $\mathscr{J}_{2}(X, \operatorname{BCC}(Y), \varphi)$ is $\varphi$-stable with respect to $\Phi$, where

$$
\Phi(x):= \begin{cases}\frac{L}{1-L} \varphi(x, 0) & \text { if (1) holds; } \\ \frac{1}{1-L} \varphi(x, 0) & \text { if (2) holds; }\end{cases}
$$

for all $x \in X$. In addition, if $f \in \mathscr{J}_{2}(X, \operatorname{BCC}(Y), \varphi)$ and there exist positive real numbers $M$ and $\alpha$ such that: $\operatorname{diam} f(x) \leq M\|x\|^{\alpha}$ for all $x \in X$, where $\alpha \in(0,1)$ if $(1)$ holds; or $\alpha \in(1, \infty)$ if $(2)$ holds, then the set-valued function $F$ given by Remark 1.3 is single-valued.

Proof. Let $(G, \circ):=(X,+)$ and $(H, *, d):=(\operatorname{BCC}(Y), \oplus, \mathcal{H})$. We define $\psi: G \times G \rightarrow \mathbb{R}_{+}$by $\psi(x, y):=\varphi(2 x, 2 y)$ for all $x, y \in G$. It is easy to see that

- if (1) holds, then $\psi\left(2^{k} x, 2^{k} y\right) \leq(2 L)^{k+1} \varphi(x, y)$ for all $x, y \in G$ and all $k \in \mathbb{N}_{0}$;

- if (2) holds, then $\psi\left(\frac{x}{2^{k}}, \frac{y}{2^{k}}\right) \leq(L / 2)^{k-1} \varphi(x, y)$ for all $x, y \in G$ and all $k \in \mathbb{N}_{0}$.

It follows that $(G, H, \psi)$ satisfies Condition (Ki) if (i) holds where $\mathrm{i}=1,2$. It follows from Theorem 2.6 that the class $\mathscr{J}_{2}(X, \operatorname{BCC}(Y), \varphi)$ is $\varphi$-stable with $\Phi$.

Final remark: The stability of set-valued functional equations inherited by that of the singlevalued corresponding equations on appropriate structures.

\section{ACKNOWLEDGEMENT}

The authors would like to thank the referee for their comments and suggestions which improve the presentation of the paper. The first author would like to thank the Development and Promotion for Science and Technology talents project (DPST) for the financial support to this paper. The second author is supported by the Thailand Research Fund and Khon Kaen University under grant RSA6280002 and Research Center for Environmental and Hazardous Substance Management, Khon Kaen University, Khon Kaen, 40002, Thailand.

\section{REFERENCES}

[1] T. Aoki: On the stability of the linear transformation in Banach spaces. J. Math. Soc. Japan 2 (1950), 64-66.

[2] K. J. Arrow, G. Debreu: Existence of an equilibrium for a competitive economy. Econometrica 22 (1954), $265-290$.

[3] R. J. Aumann: Integrals of set-valued functions. J. Math. Anal. Appl. 12 (1965), 1-12.

[4] J. Brzdęk: Stability of additivity and fixed point methods. Fixed Point Theory Appl. 2013 (2013), Article ID 401756, 9 pages.

[5] C. Castaing, M. Valadier: Convex analysis and measurable multifunctions: Lec. Notes in Math. Springer, Berlin, 1977.

[6] J. B. Diaz, B. Margolis: A fixed point theorem of the alternative for contractions on a generalized complete metric space. Bull. Amer. Math. Soc. 74 (1968), 305-309.

[7] G. L. Forti: An existence and stability theorems for a class of functional equations. Stochastica 4 (1) (1980), $23-30$.

[8] Z. Gajda: On stability of additive mappings. Internat. J. Math. Math. Sci. 14(3) (1991), 431-434.

[9] P. Găvruța: A generalization of the Hyers-Ulam-Rassias stability of approximately additive mappings. J. Math. Anal. Appl. 184(3) (1994), 431-436.

[10] D. H. Hyers: On the stability of the linear functional equation. Proc. Nat. Acad. Sci. U.S.A. 27 (1941), $222-224$.

[11] G. H. Kim: Addendum to 'On the stability of functional equations on square-symmetric groupoid. Nonlinear Anal. 62 (2005), 365-381.

[12] Z. Páles, P. Volkman and R. D. Luce: Hyers-Ulam stability of functional equations with a square-symmetric operation. Proc. Natl. Acad. Sci. USA, 95 (1998), 12772-12275.

[13] C. Park, S. Yun, J. Lee and D. Shin: Set-valued additive functional equations. Constr. Math. Anal. 2(2) (2019), 89-97.

[14] H. Przybycień: A note on closedness of algebraic sum of sets. Tbilisi Math. J. 9 (2) (2016), 71-74.

[15] Th. M. Rassias: On the stability of the linear mapping in Banach spaces. Proc. Amer. Math. Soc. 72 (1978), $297-300$.

[16] S. Saejung, J. Senasukh: On stability and hyperstability of additive equations on a commutative semigroup. Acta Math. Hungar. 159(2) (2019), 358-373.

[17] S. M. Ulam: A collection of mathematical problems. Interscience Publishers, New York-London, 1960. 
Department of Mathematics, Faculty of SCience, KHON KAEN UniVERSity, KHON KAEN, 40002, ThaILAND ORCID: 0000-0002-5264-8728

E-mail address: senasukh@kkumail.com

Department of Mathematics, Faculty of SCience, KhON Kaen University, KHON KaEN 40002, Thailand; Research CENTER For ENVIRONMENTAl and HaZardous Substance Management (EHSM), KHON KAEN University, KHON KAEN 40002, ThaILAND; AND Center of Excellence on Hazardous Substance Management (HSM), PATUMWAN, BANGKOK, 10330, THAILAND

ORCID: 0000-0003-3325-2864

E-mail address: saejung@kku.ac.th 